\title{
Association of Speech Recognition Thresholds With Brain Volumes and White Matter Microstructure: The Rotterdam Study
}

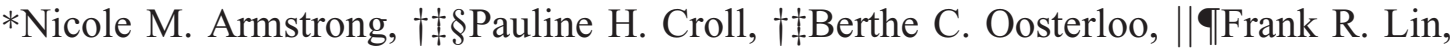 \\ $\ddagger$ M. Arfan Ikram, †André Goedegebure, and $\ddagger \S$ Meike W. Vernooij
}

*Laboratory of Behavioral Neuroscience, National Institute on Aging, Baltimore, Maryland, USA; †Department of Otorhinolaryngology, Head and Neck Surgery; †Department of Epidemiology; §Department of Radiology and Nuclear Medicine, Erasmus University Medical Center, Rotterdam, the Netherlands; ||Department of Otolaryngology—Head and Neck Surgery, Johns Hopkins University Medical School; and $\mathbf{9 D e p a r t m e n t s ~ o f ~ E p i d e m i o l o g y ~ a n d ~ M e n t a l ~ H e a l t h , ~ J o h n s ~ H o p k i n s ~ B l o o m b e r g ~ S c h o o l ~ o f ~}$ Public Health, Baltimore, Maryland, USA

Objectives: Brain volumetric declines may underlie the association between hearing loss and dementia. While much is known about the peripheral auditory function and brain volumetric declines, poorer central auditory speech processing may also be associated with decreases in brain volumes. Methods: Central auditory speech processing, measured by the speech recognition threshold (SRT) from the Digits-inNoise task, and neuroimaging assessments (structural magnetic resonance imaging [MRI] and fractional anisotropy and mean diffusivity from diffusion tensor imaging), were assessed cross-sectionally in 2,368 Rotterdam Study participants aged 51.8 to 97.8 years. SRTs were defined continuously and categorically by degrees of auditory performance (normal, insufficient, and poor). Brain volumes from structural MRI were assessed on a global and lobar level, as well as for specific dementia-related structures (hippocampus, entorhinal cortex, parahippocampal gyrus). Multivariable linear regression models adjusted by age, age-squared, sex, educational level, alcohol consumption, intracranial volume
(MRI only), cardiovascular risk factors (hypertension, diabetes, obesity, current smoking), and pure-tone average were used to determine associations between SRT and brain structure.

Results: Poorer central auditory speech processing was associated with larger parietal lobe volume (difference in $\mathrm{mL}$ per $\mathrm{dB}$ increase $=0.24,95 \% \mathrm{CI}$ : $0.05,0.42$ ), but not with diffusion tensor imaging measures. Degrees of auditory performance were not associated with brain volumes and white matter microstructure.

Conclusions: Central auditory speech processing in the presence of both vascular burden and pure-tone average may not be related to brain volumes and white matter microstructure. Longitudinal follow-up is needed to explore these relationships thoroughly. Key Words: Brain volumesCentral auditory speech processing-Digits-in-noise testWhite matter.

Otol Neurotol 41:xxx-xxx, 2020.
Address correspondence and reprint requests to André Goedegebure, Ph.D., Department of Otorhinolaryngology, Head and Neck Surgery, Erasmus MC, P.O. Box 2040, 3000 CA Rotterdam, the Netherlands; E-mail: a.goedegebure@erasmusmc.nl

N.M.A. wrote the manuscript and analyzed the data. All authors discussed the results and implications and commented on the manuscript at all stages, and they were responsible for the study design.

The Rotterdam Study is supported by the Erasmus Medical Center and Erasmus University, Rotterdam, The Netherlands; the Organization for Scientific Research; the Netherlands Organization for Health Research and Development; the Research Institute for Diseases in the Elderly; the Netherlands Genomics Initiative; the Ministry of Education, Culture, and Science; the Ministry of Health, Welfare, and Sports; the European Commission (DG XII); and the Municipality of Rotterdam. Hearing-related research within the Rotterdam Study is partly funded by The Heinsius Houbolt Foundation. Separately, N.M.A. is supported by the Intramural Research Program at the National Institute on Aging, Baltimore, Maryland (N.M.A. received funding).

F.R.L., A.G., and P.H.C. report funding by Cochlear Ltd.

The authors disclose no conflicts of interest.

Supplemental digital content is available in the text.

DOI: 10.1097/MAO.0000000000002739
Age-related hearing loss is a prevalent condition affecting up to two-thirds of adults aged 70 years and older (1). Recently, it has been recognized as being a potentially modifiable risk factor of dementia (2). While there have been multiple studies examining objective peripheral auditory function and its relationship to dementia and brain health, there is still a dearth of studies examining central auditory speech processing and its relationship to the brain. Peripheral auditory function reflects the encoding of sound into a neural signal in the cochlea, and central auditory speech processing reflects the further processing and decoding of this neural signal in the brain, which is dependent on multiple regions in the cortex and brainstem for the way in which the brain comprehends sounds outside of the cochlea $(3,4)$.

Speech-in-noise tests can serve as a measure of auditory performance encompassing components of both central and peripheral auditory function. These tests are largely covered by pure-tone audiometry, and they 
require substantial attentional and behavioral processing. Speech-in-noise tests, based on spoken sentences, may not be a good indicator of the ability of understanding speech through noise, since some people cannot understand and repeat complete sentences in noise, regardless of degree of hearing loss. This test could be confounded by elements, such as memory and linguistic aspects, instead of hearing loss separately. One particular test that has been developed is the Digits-In-Noise (DIN) test, a test that requires individuals to repeat three spoken numbers through concurrent background noise presented to the ear (5) and relies on minimal language skills to do so. The correlation between tests of sentence comprehension and DIN test is very high, so both primarily measure the ability to understand speech in noise (6).

We aimed to examine the association of central auditory speech processing-as assessed with DIN and adjusted by a measure of peripheral auditory function, pure-tone average - with brain volumes and white matter (WM) microstructure, measured with MRI and diffusion tensor imaging (DTI) respectively, in a population-based cohort study. We hypothesize that poorer central auditory speech processing is associated with smaller brain volumes and decreased WM microstructure among cognitively normal older adults. As a further step, we evaluated the associations of the degrees of auditory performance (normal, insufficient, and poor) from the DIN test with structural brain parameters to determine whether poor and insufficient auditory performance is related to smaller brain volumes and less WM microstructure than normal auditory performance.

\section{METHODS}

\section{Participants}

This cross-sectional study was embedded in the Rotterdam Study, a prospective cohort study of determinants and consequences of aging (7). Enrollment criteria are described in more detail elsewhere (7). Briefly, from the population registry of Ommoord, a suburb of Rotterdam, The Netherlands, adults aged $\geq 45$ years were invited to participate in the Rotterdam Study. Enrollment took place on a voluntary basis. Since 2005, all Rotterdam Study participants undergo brain MRI as part of the study protocol. In 2011, hearing assessments were introduced into the study protocol. There were 3,667 cognitively normal participants with both completed pure-tone audiometry and DIN. We excluded individuals with greater than two standard deviations from the mean speech recognition thresholds of DIN $(n=169)$. This left 2,386 participants with completed hearing and magnetic resonance assessments that occurred within the same year. Of these participants, 2,226 participants also had good quality diffusion tensor imaging data available. Details on the determination of cognitive status is described elsewhere (8). The institutional review board (Medical Ethics Committee) and the review board of The Netherlands Ministry of Health, Welfare, and Sports approved this study, and participants provided written informed consent.

\section{Audiometry}

All audiometry was performed in a soundproof booth by a single qualified healthcare professional. A clinical audiometer (Decos audiology workstation, version 210.2.6, with AudioNigma interface), TDH-39P earphones, and B71 bone conductor were used.

Pure-tone audiometry thresholds were measured according to the International Organization for Standardization 8253-1 (International Organization for Standardization, 2010). Air conduction $(0.25,0.50,1,2,4$, and $8 \mathrm{kHz}$ ) and bone conduction (only two frequencies due to limited time: 0.5 and $4 \mathrm{kHz}$ ) were tested for both the ears. Masking was done according to the method of Hood (1960). We calculated the average threshold over all frequencies for both ears. If both the ears were equal, right or left was alternately chosen. We used the average threshold over all frequencies for the better ear.

Additionally, the DIN test was performed to detect the speech recognition ability in noise for the best hearing ear (9). The test measures a speech reception threshold (SRT) by letting participants repeat digit triplets in an automated adaptive procedure and changing the signal-tonoise ratio according to the correctness of the answer. The SRT represents a speech-in-noise ratio for 50\% correctly repeated triplets. A higher value represents a worse ability of understanding speech in noise.

We defined degrees of auditory performance from DIN summary scores. The categories are: normal $(\leq-5.55 \mathrm{~dB}$ signal-to-noise ratio [SNR]), insufficient $(>-5.55$ to $\leq 3.80 \mathrm{~dB})$, and poor $(>-3.80 \mathrm{~dB}$ SNR). These values are similar to the ones reported by Dawes et al. (10).

\section{MRI Acquisition and Processing}

Multisequence MRI imaging was performed in a 1.5 Tesla MRI scanner (GE Signa Excite; GE Healthcare, Milwaukee, USA). The scan protocol and sequence details have been described elsewhere (11).

For volumetry, T1-weighted (voxel size $0.49 \times 0.49 \times 1.6 \mathrm{~mm}^{3}$ ), proton density-weighted (voxel size $\left.0.6 \times 0.98 \times 1.6 \mathrm{~mm}^{3}\right)$, and the fluid-attenuated inversion recovery (voxel size $0.78 \times 1.12 \times 2.5 \mathrm{~mm}^{3}$ ) scans were used for automated segmentation of supratentorial gray matter (GM), WM, cerebrospinal fluid (CSF), and WM lesions $(12,13)$. All scans were transformed to the high-resolution data set $(256 \times 256 \times 128)$ using trilinear interpolation. Automated processing tools from the Brain Imaging Center, Montreal Neurological Institute, and McGill University (www.bic.mni.mcgill.ca) were used to coregister the MRI data and subsequently normalize the intensities for each feature image volume using N3 (14). All segmentations were visually inspected and manually corrected on an as-needed basis.

We examined 19 global and lobar brain regions of interest for volumetry. For global regions, we included total brain volume, total GM, and total WM. Total brain volume was the sum of GM, normal-appearing WM, and WM lesion volume. Intracranial volume was estimated by summing GM and WM (consisting of the sum of normal-appearing WM and WM lesion volume) and CSF 
volumes (12). For lobar regions, an atlas was created in which the lobes were labeled, according to a slightly modified version of the segmentation protocol $(15,16)$. Nonrigid transformation was used to transform this atlas to each brain to obtain each lobe volume (16). For specific regions of interest, T1-weighted MR images were processed using FreeSurfer version 5.1 (17) to obtain cortical parcellations and subcortical structural volume of the hippocampus, entorhinal cortex, and parahippocampal gyrus, regions affected by early Alzheimer disease pathology (18-22).

For microstructural measures, DTI (voxel size $3.3 \times 2.2 \times 3.5 \mathrm{~mm}^{3}$ ) was used. DTI is MRI technique that can map and characterize the three-dimensional diffusion of water as a function of spatial location $(23,24)$. A single shot, diffusion-weighted spin echo echo-planar imaging sequence was performed with maximum $b$ value of $1,000 \mathrm{~s} / \mathrm{mm}^{2}$ in 25 noncollinear directions; 3 bo volumes were acquired without diffusion weighting. Diffusion data were preprocessed using a standardized processing pipeline (25). DTI measures include fractional anisotropy (FA), a measure of the directionality of water diffusion, and mean diffusivity (MD), a measure of the magnitude of water diffusion within brain tissue. Global mean FA and MD were computed from the normal-appearing white matter and ventricles (CSF). In WM, axons, myelin sheaths, and neurofilaments restrict both direction and magnitude of diffusion, leading to highly directional diffusion running in parallel with the WM structures. This is associated with high levels of FA in the WM, compared with cerebral gray matter (26). As WM structures become damaged, lower FA is expected as the directional order imposed is lost and higher MD is expected as there are less barriers restricting the overall magnitude of diffusion.

\section{Covariates}

Demographic characteristics (age, sex, educational level), vascular burden, and alcohol consumption were covariates that were collected through a home interview at study enrollment or by recurrent physical examination and blood sampling at study center (7). Age was the age at the concurrent hearing and MRI assessments. Educational level was categorized as having completed primary level, secondary level, or higher education. Vascular burden was defined by a sum of the following cardiovascular risk factors: elevated total cholesterol $(\geq 200 \mathrm{mg} / \mathrm{dL})$, hypertension (systolic blood pressure $\geq 140 \mathrm{~mm} \mathrm{Hg}$, diastolic blood pressure $\geq 90 \mathrm{~mm} \mathrm{Hg}$, and/or use of antihypertensive medications), diabetes (fasting glucose of $\geq 7 \mathrm{mmol} / \mathrm{L}$, nonfasting glucose $\geq 11 \mathrm{mmol} / \mathrm{L}$, or use of diabetes medications), self-reported smoking status (current smoker versus never or former smoker), and obesity $(\geq 30 \mathrm{~kg} /$ $\left.\mathrm{m}^{2}\right)(27)$. We further categorized vascular burden into the following: 0 for the absence of cardiovascular risk factors, 1 for one cardiovascular risk factor, and $\geq 2$ for two or more cardiovascular risk factors. Additionally, alcohol consumption was defined as number of grams consumed per day, on average, as assessed with a validated, self- administered, semiquantitative food-frequency questionnaire $(28,29)$.

\section{Statistical Analysis}

Chi-squared tests were used for categorical variables, while ANOVAs were used for continuous variables to calculate differences in sample characteristics. We used two multivariable linear regression models. We regressed brain volumes and WM microstructure on SRT, age, agesquared, and sex for the first model (Model 1). For the second model (Model 2), we regressed brain volumes and WM microstructure on SRT, age, age-squared, sex, educational level, alcohol consumption, and vascular burden sum score categories $(0,1,2+$ cardiovascular risk factors). Age-squared was added as term in both models to adjust for the nonlinear estimated effects of age on brain volumes and WM microstructure. ICV was included as additional adjustment covariate for analyses of volumetry. We also present the models with adjustment from pure-tone average (PTA) of all frequencies as main analyses and without PTA adjustment for SRTdefined categories of auditory performance as a supplemental table, http://links.lww.com/MAO/B21. PTA of all frequencies was used, since there could be highfrequency hearing loss associated with changes in brain volumes and WM microstructure. The variability of SRT explained by PTA after age and sex adjustment was $54 \%$ in this sample, so PTA may confound the association of SRT with brain volumes and WM microstructure. Associations were considered significant if the $95 \%$ confidence intervals did not overlap with 0 .

\section{Data Availability}

Data can be obtained on request. Requests should be directed toward the management team of the Rotterdam Study (secretariaat.epi@erasmusmc.nl),which has a protocol for approving data requests. Because of restrictions based on national and European privacy regulations and informed consent of the participants, data cannot be made freely available in a public repository.

\section{RESULTS}

\section{Characteristics of Study Sample}

Table 1 shows the characteristics of the study sample $(\mathrm{n}=2,386)$. The mean age of the participants was 64.8 $(\mathrm{SD}=7.1)$ years, ranging from 51.8 to 97.8 years. There were $1,096(45.9 \%)$ males. The average SRT was -4.2 $(\mathrm{SD}=4.1)$ decibels, and the average PTA in the better ear was 22.3 (11.0) decibels. Approximately 46.4\% of the sample $(\mathrm{n}=1,106)$ had at least one cardiovascular risk factor. About $21.0 \%$ of the sample $(n=501)$ was obese, and about $63.8 \%$ of the sample $(n=1,520)$ had hypertension. Approximately $37.8 \%(\mathrm{n}=926)$ reported use of antihypertensive medications, and $28.7 \% \quad(\mathrm{n}=701)$ reported use of lipid-lowering medications.

There were differences across degrees of auditory performance. Those with moderate or greater hearing loss were, on average, older with lower SRT scores, 
TABLE 1. Characteristics of participants in the Rotterdam study $(n=2,368)$

\begin{tabular}{|c|c|c|c|c|c|}
\hline \multirow[b]{2}{*}{ Baseline Characteristics } & \multirow[b]{2}{*}{$\begin{array}{l}\text { Overall Sample } \\
\quad(\mathrm{n}=2,386)\end{array}$} & \multicolumn{3}{|c|}{ Degree of Auditory Performance } & \multirow{2}{*}{$\begin{array}{c}p \text { Value } \\
\text { for the } \\
\text { Difference }\end{array}$} \\
\hline & & $\begin{array}{c}\text { Normal } \\
(\mathrm{n}=1,138)\end{array}$ & $\begin{array}{l}\text { Insufficient } \\
(\mathrm{n}=554)\end{array}$ & $\begin{array}{c}\text { Poor } \\
(\mathrm{n}=694)\end{array}$ & \\
\hline Age, in years, mean (SD) & $64.8(7.1)$ & $62.3(5.7)$ & $64.9(6.2)$ & $68.9(7.7)$ & $<0.001$ \\
\hline Males, n (\%) & $1.096(45.9)$ & $472(41.5)$ & $256(46.2)$ & $368(53.0)$ & $<0.001$ \\
\hline Educational level, n (\%) & & & & & $<0.001$ \\
\hline Primary education & $168(7.0)$ & $55(4.8)$ & $39(7.0)$ & $74(10.7)$ & \\
\hline $\begin{array}{l}\text { Lower/intermediate general education } \\
\text { or lower vocational education }\end{array}$ & $822(34.5)$ & $378(33.2)$ & $192(34.7)$ & $252(36.3)$ & \\
\hline $\begin{array}{l}\text { Intermediate vocational education or } \\
\text { higher general education }\end{array}$ & $730(30.6)$ & $346(30.4)$ & $163(29.4)$ & $221(31.8)$ & \\
\hline $\begin{array}{l}\text { Higher vocational education or } \\
\text { university }\end{array}$ & $650(27.2)$ & $354(31.1)$ & $158(28.5)$ & $138(19.9)$ & \\
\hline $\begin{array}{l}\text { Speech recognition threshold scores, } \\
\text { mean (SD) }\end{array}$ & $-4.2(4.1)$ & $-6.7(0.8)$ & $-4.8(0.5)$ & $0.5(4.8)$ & $<0.001$ \\
\hline $\begin{array}{l}\text { Pure-tone average in the better ear, } \\
\text { in decibels, mean (SD) }\end{array}$ & $22.3(11.0)$ & $15.7(6.3)$ & $21.5(6.9)$ & $34.1(10.2)$ & $<0.001$ \\
\hline Vascular burden, n (\%) & & & & & $<0.001$ \\
\hline No cardiovascular risk factors & $698(29.3)$ & $412(36.2)$ & $156(28.2)$ & $130(18.7)$ & \\
\hline 1 cardiovascular risk factor & $1,106(46.4)$ & $510(44.8)$ & $256(46.2)$ & $340(49.0)$ & \\
\hline $2+$ cardiovascular risk factors & $582(24.4)$ & $216(19.0)$ & $142(25.6)$ & $224(32.3)$ & \\
\hline Hypertension, n (\%) & $1.520(63.8)$ & $643(56.5)$ & $353(63.7)$ & $524(75.5)$ & $<0.001$ \\
\hline Elevated total cholesterol, $\mathrm{n}(\%)$ & - & - & - & - & \\
\hline Diabetes, n (\%) & $203(8.5)$ & $61(5.4)$ & $56(10.1)$ & $86(12.4)$ & $<0.001$ \\
\hline Current smoking status, $\mathrm{n}(\%)$ & $152(6.4)$ & $54(4.8)$ & $42(7.6)$ & $56(8.1)$ & $<0.001$ \\
\hline Obesity, n (\%) & $501(21.0)$ & $220(19.3)$ & $118(21.3)$ & $163(23.5)$ & 0.005 \\
\hline $\begin{array}{l}\text { Use of antihypertensive medications, } \\
\mathrm{n}(\%)\end{array}$ & $926(37.8)$ & $385(33.2)$ & $210(36.8)$ & $331(46.3)$ & $<0.001$ \\
\hline $\begin{array}{l}\text { Use of lipid-lowering medications, } \\
\mathrm{n}(\%)\end{array}$ & $701(28.7)$ & $288(24.8)$ & $166(29.1)$ & $247(34.6)$ & $<0.001$ \\
\hline $\begin{array}{l}\text { Systolic blood pressure, in } \mathrm{mm} \mathrm{Hg} \text {, } \\
\text { mean (SD) }\end{array}$ & $144.5(21.6)$ & $141.1(21.6)$ & $143.8(20.9)$ & $148.7(21.5)$ & $<0.001$ \\
\hline $\begin{array}{l}\text { Diastolic blood pressure, in } \mathrm{mm} \mathrm{Hg} \text {, } \\
\text { mean (SD) }\end{array}$ & $84.0(11.5)$ & $83.1(11.5)$ & $84.3(11.6)$ & $84.9(11.5)$ & 0.070 \\
\hline $\begin{array}{l}\text { Total cholesterol, in } \mathrm{mmol} / \mathrm{L} \text {, } \\
\text { mean }(\mathrm{SD})\end{array}$ & $5.5(1.1)$ & $5.6(1.1)$ & $5.5(1.1)$ & $5.4(1.1)$ & 0.003 \\
\hline $\begin{array}{l}\text { Daily alcohol consumption, in grams, } \\
\text { mean (SD) }\end{array}$ & $13.0(15.6)$ & $13.4(15.0)$ & $11.8(15.8)$ & $13.2(16.4)$ & 0.210 \\
\hline $\begin{array}{l}\text { Intracranial volume, in } \mathrm{mL} \text {, } \\
\text { mean (SD) }\end{array}$ & $1,140,454(112,517.7)$ & $1,142,398(112,095.6)$ & $1,132,780(112,451.8)$ & $1,143,409(113,148.4)$ & 0.184 \\
\hline
\end{tabular}

Comparisons across hearing categories were done using $\chi^{2}$ tests for categorical variables and ANOVA for continuous variables, and $p$ values for the difference are recorded in the table. The hearing status categories were defined by the cut-points from the speech recognition thresholds: normal $(\leq-5.55 \mathrm{~dB})$, insufficient $(-5.55$ to $-3.80 \mathrm{~dB})$, and poor $(>-3.80 \mathrm{~dB})$. There were some variables missing observations. These were: education $(n=16)$, pure-tone average $(n=33)$, hypertension $(n=2)$, diabetes $(n=1)$, smoking status $(n=1,054)$, obesity $(n=46)$, use of antihypertensive treatment and lipid-lowering medications $(\mathrm{n}=5)$, systolic and diastolic blood pressure $(\mathrm{n}=1,252)$, alcohol consumption $(n=462)$, and total cholesterol $(n=28)$.

$\mathrm{SD}$ indicates standard deviation.

greater pure-tone averages, higher alcohol consumption, and higher systolic blood pressure than those with mild hearing loss or normal hearing (Table 1). Those with moderate or greater hearing loss were more likely to be male and have at least one cardiovascular risk factor (diabetes, current smoking status, obesity, hypertension), and use of antihypertensive and lipid-lowering medications, as compared with those with mild hearing loss and normal hearing (Table 1).

\section{Associations of Speech Recognition Thresholds With Brain Volumes}

We examined the associations of the SRTs continuously and categorically with brain volumes in each 
TABLE 2. Association of continuous speech recognition thresholds with brain volumes in the Rotterdam study $(n=2,368)$

\begin{tabular}{|c|c|c|c|c|c|c|c|c|}
\hline \multirow[b]{3}{*}{$\begin{array}{l}\text { Brain Regions of } \\
\text { Interest }\end{array}$} & \multicolumn{4}{|c|}{ Model 1} & \multicolumn{4}{|c|}{ Model 2} \\
\hline & \multicolumn{2}{|c|}{ Unadjusted for PTA } & \multicolumn{2}{|c|}{ Adjusted for PTA } & \multicolumn{2}{|c|}{ Unadjusted for PTA } & \multicolumn{2}{|c|}{ Adjusted for PTA } \\
\hline & $\begin{array}{l}\text { Difference } \\
\text { in } \mathrm{mL} \\
\text { of Brain } \\
\text { Volumes }\end{array}$ & $\begin{array}{c}95 \% \\
\text { Confidence } \\
\text { Interval }\end{array}$ & $\begin{array}{l}\text { Difference } \\
\text { in mL of } \\
\text { Brain } \\
\text { Volumes }\end{array}$ & $\begin{array}{c}95 \% \\
\text { Confidence } \\
\text { Interval }\end{array}$ & $\begin{array}{l}\text { Difference } \\
\text { in } \mathrm{mL} \\
\text { of Brain } \\
\text { Volumes }\end{array}$ & $\begin{array}{c}95 \% \\
\text { Confidence } \\
\text { Interval }\end{array}$ & $\begin{array}{l}\text { Difference } \\
\text { in mL of } \\
\text { Brain } \\
\text { Volumes }\end{array}$ & $\begin{array}{c}95 \% \\
\text { Confidence } \\
\text { Interval }\end{array}$ \\
\hline Total brain & -0.20 & $(-0.56,0.17)$ & 0.25 & $(-0.30,0.80)$ & 0.00 & $(-0.39,0.39)$ & 0.58 & $(-0.01,1.18)$ \\
\hline GM & 0.04 & $(-0.29,0.37)$ & 0.05 & $(-0.45,0.55)$ & 0.18 & $(-0.18,0.54)$ & 0.32 & $(-0.23,0.87)$ \\
\hline WM & -0.27 & $(-0.65,0.12)$ & 0.10 & $(-0.48,0.67)$ & -0.19 & $(-0.61,0.23)$ & 0.20 & $(-0.43,0.83)$ \\
\hline Temporal lobe & -0.04 & $(-0.13,0.05)$ & 0.05 & $(-0.08,0.19)$ & 0.00 & $(-0.10,0.10)$ & 0.09 & $(-0.06,0.24)$ \\
\hline Temporal GM & -0.01 & $(-0.09,0.06)$ & -0.02 & $(-0.14,0.09)$ & 0.02 & $(-0.06,0.11)$ & 0.01 & $(-0.12,0.14)$ \\
\hline Temporal WM & -0.03 & $(-0.10,0.04)$ & 0.04 & $(-0.07,0.15)$ & -0.01 & $(-0.09,0.07)$ & 0.07 & $(-0.05,0.19)$ \\
\hline Parietal lobe & -0.04 & $(-0.15,0.07)$ & 0.12 & $(-0.05,0.29)$ & 0.03 & $(-0.09,0.15)$ & 0.24 & $(0.05,0.42)$ \\
\hline Parietal GM & 0.02 & $(-0.06,0.10)$ & 0.04 & $(-0.08,0.16)$ & 0.05 & $(-0.04,0.14)$ & 0.11 & $(-0.02,0.24)$ \\
\hline Parietal WM & -0.04 & $(-0.14,0.05)$ & 0.05 & $(-0.09,0.20)$ & 0.00 & $(-0.10,0.11)$ & 0.11 & $(-0.04,0.27)$ \\
\hline Frontal lobe & -0.06 & $(-0.24,0.12)$ & 0.15 & $(-0.12,0.42)$ & 0.00 & $(-0.19,0.19)$ & 0.23 & $(-0.06,0.52)$ \\
\hline Frontal GM & 0.02 & $(-0.10,0.15)$ & 0.07 & $(-0.12,0.26)$ & 0.07 & $(-0.07,0.20)$ & 0.14 & $(-0.06,0.35)$ \\
\hline Frontal WM & -0.06 & $(-0.21,0.09)$ & 0.07 & $(-0.15,0.29)$ & -0.03 & $(-0.19,0.13)$ & 0.12 & $(-0.12,0.36)$ \\
\hline Occipital lobe & 0.02 & $(-0.06,0.10)$ & 0.00 & $(-0.12,0.12)$ & 0.02 & $(-0.06,0.11)$ & 0.02 & $(-0.11,0.15)$ \\
\hline Occipital GM & 0.03 & $(-0.03,0.09)$ & 0.03 & $(-0.06,0.12)$ & 0.04 & $(-0.03,0.10)$ & 0.05 & $(-0.05,0.15)$ \\
\hline Occipital WM & -0.02 & $(-0.08,0.04)$ & -0.03 & $(-0.12,0.06)$ & -0.01 & $(-0.08,0.05)$ & -0.02 & $(-0.12,0.08)$ \\
\hline Hippocampus & 0.00 & $(-0.01,0.01)$ & 0.00 & $(-0.01,0.01)$ & 0.00 & $(-0.01,0.01)$ & 0.00 & $(-0.01,0.02)$ \\
\hline Entorhinal cortex & 0.00 & $(0.00,0.00)$ & 0.00 & $(0.00,0.00)$ & 0.00 & $(0.00,0.00)$ & 0.00 & $(0.00,0.00)$ \\
\hline Parahippocampal gyrus & 0.00 & $(0.00,0.00)$ & 0.00 & $(0.00,0.00)$ & 0.00 & $(0.00,0.00)$ & 0.00 & $(0.00,0.00)$ \\
\hline
\end{tabular}

Note: Model 1 was a multivariable linear regression model adjusted by age, age-squared, intracranial volume, and sex. Model 2 was a multivariable linear regression model adjusted by age, age-squared, sex, educational level, alcohol consumption, intracranial volume, and vascular burden sum score categories $(0,1,2+$ cardiovascular risk factors). All continuous variables (age, intracranial volume, alcohol consumption, and pure-tone average) were mean-centered. Bolded values indicate that the $95 \%$ confidence interval does not overlap with 0 .

CSF indicates cerebrospinal fluid; GM, gray matter; PTA, pure-tone average; WM, white matter.

model. After PTA adjustment, there were no associations in Model 1, but there were associations of higher SRTs with higher parietal lobe volume (difference in $\mathrm{mL}=0.24,95 \% \mathrm{CI}: 0.05,0.42$ ) in Model 2 (Table 2). Before PTA adjustment, there were no associations between continuous SRTs and brain volumes in both models (Table 2).

When examining the association between degrees of auditory performance (normal, insufficient, and poor) and brain volumes cross-sectionally after PTA adjustment, degrees of auditory performance were not associated with brain volumes (Table 3). Before PTA adjustment, those with poor auditory performance had lower volumes in total brain (difference in $\mathrm{mL}=-3.53,95 \% \mathrm{CI}:-6.89,-0.16$ ) and $\mathrm{WM}$ volume (difference in $\mathrm{mL}=-4.42,95 \% \mathrm{CI}$ : $-7.95,-0.88$ ), particularly in frontal (difference in $\mathrm{mL}=-1.08,95 \% \mathrm{CI}:-1.95,-0.20)$ and parietal $\mathrm{WM}$ (difference in $\mathrm{mL}=-1.76,95 \% \mathrm{CI}$ : $-3.12,-0.41$ ), as compared with those with normal hearing (Supplemental Table 1, http://links.lww.com/MAO/B21). In Model 2, poor auditory performance was associated with smaller volumes in $\mathrm{WM}$ (difference in $\mathrm{mL}=-4.40,95 \% \mathrm{CI}$ : $-8.39,-0.42$ ), especially in frontal WM (difference in $\mathrm{mL}=-1.76,95 \% \mathrm{CI}:-3.27,-0.25$ ) (Supplemental Table 1, http://links.lww.com/MAO/B21).

Regarding the relationship between continuous SRT and laterality of brain volumes, there were no differences in the results by hemisphere. In terms of the relationship between SRT-defined degrees of auditory performance and laterality of brain volumes, there were no associations between insufficient auditory performance and brain volumes in either model. For Model 1, poor auditory performance was associated with lower left frontal WM volumes (difference in $\mathrm{mL}=-0.92,95 \% \mathrm{CI}:-1.81,-0.03$ ), but this association was not found in Model 2.

\section{Association of Speech Recognition Thresholds With White Matter Microstructure}

We examined the associations of the SRTs continuously and categorically with global measures of WM microstructure, MD, and FA. There were no associations of SRTs categorically and continuously with MD in any of the models (Table 4). After PTA adjustment, there were no associations of continuous SRT with FA in Models 1 and 2 (Table 4). Before PTA adjustment, higher SRT was associated with lower FA (difference $=-0.0019 \times 10^{-2}, 95 \% \mathrm{CI}$ : -0.0036 $\times 10^{-2},-0.0002 \times 10^{-2}$ ) in Model 1, but no associations were present in Model 2 (Table 3). When examining the associations of SRT-defined degrees of auditory performance with FA and MD, we found that insufficient auditory performance was associated with lower FA (difference $=-0.0196$ $\left.\times 10^{-2}, 95 \% \mathrm{CI}:-0.0354 \times 10^{-2},-0.0039 \times 10^{-2}\right)$ in Model 1 after PTA adjustment. There was a marginally significant association between poor auditory performance and lower FA in Model 1. Before PTA adjustment, insufficient auditory performance was associated with lower FA 
TABLE 3. Association of degrees of auditory performance defined by speech recognition thresholds with brain volumes adjusted by pure-tone average in the Rotterdam study $(n=2,368)$

\begin{tabular}{|c|c|c|c|c|c|c|c|c|}
\hline \multirow[b]{3}{*}{$\begin{array}{l}\text { Brain Regions of } \\
\text { Interest }\end{array}$} & \multicolumn{4}{|c|}{ Model 1} & \multicolumn{4}{|c|}{ Model 2} \\
\hline & \multicolumn{2}{|c|}{$\begin{array}{c}\text { Insufficient Versus Normal } \\
\text { Auditory Performance }\end{array}$} & \multicolumn{2}{|c|}{$\begin{array}{l}\text { Poor versus Normal } \\
\text { Auditory Performance }\end{array}$} & \multicolumn{2}{|c|}{$\begin{array}{c}\text { Insufficient versus Normal } \\
\text { Auditory Performance }\end{array}$} & \multicolumn{2}{|c|}{$\begin{array}{l}\text { Poor Versus Normal } \\
\text { Auditory Performance }\end{array}$} \\
\hline & $\begin{array}{c}\text { Annual } \\
\text { Difference } \\
\text { in mL of } \\
\text { Brain } \\
\text { Volumes }\end{array}$ & $\begin{array}{c}95 \% \\
\text { Confidence } \\
\text { Interval }\end{array}$ & $\begin{array}{c}\text { Annual } \\
\text { Difference } \\
\text { in } \mathrm{mL} \text { of } \\
\text { Brain } \\
\text { Volumes }\end{array}$ & $\begin{array}{c}95 \% \\
\text { Confidence } \\
\text { Interval }\end{array}$ & $\begin{array}{c}\text { Annual } \\
\text { Difference } \\
\text { in } \mathrm{mL} \text { of } \\
\text { Brain } \\
\text { Volumes }\end{array}$ & $\begin{array}{c}95 \% \\
\text { Confidence } \\
\text { Interval }\end{array}$ & $\begin{array}{c}\text { Annual } \\
\text { Difference } \\
\text { in } \mathrm{mL} \text { of } \\
\text { Brain } \\
\text { Volumes }\end{array}$ & $\begin{array}{c}95 \% \\
\text { Confidence } \\
\text { Interval }\end{array}$ \\
\hline Total brain & 0.45 & $(-3.01,3.91)$ & -1.28 & $(-5.67,3.12)$ & 0.74 & $(-3.11,4.59)$ & -0.62 & $(-5.52,4.29)$ \\
\hline GM & 1.27 & $(-1.89,4.44)$ & 1.15 & $(-2.87,5.18)$ & 1.27 & $(-2.33,4.87)$ & 1.86 & $(-2.72,6.45)$ \\
\hline WM & -0.65 & $(-4.29,2.99)$ & -2.73 & $(-7.36,1.90)$ & -0.76 & $(-4.89,3.37)$ & -3.23 & $(-8.48,2.02)$ \\
\hline Temporal lobe & 0.33 & $(-0.53,1.19)$ & 0.32 & $(-0.77,1.41)$ & 0.42 & $(-0.57,1.41)$ & 0.48 & $(-0.78,1.74)$ \\
\hline Temporal GM & 0.21 & $(-0.52,0.95)$ & 0.35 & $(-0.58,1.29)$ & 0.24 & $(-0.61,1.08)$ & 0.50 & $(-0.57,1.57)$ \\
\hline Temporal WM & 0.11 & $(-0.57,0.79)$ & -0.22 & $(-1.09,0.64)$ & 0.15 & $(-0.63,0.93)$ & -0.09 & $(-1.08,0.90)$ \\
\hline Parietal lobe & -0.28 & $(-1.35,0.80)$ & -0.13 & $(-1.50,1.23)$ & -0.16 & $(-1.36,1.03)$ & 0.35 & $(-1.18,1.87)$ \\
\hline Parietal GM & 0.24 & $(-0.53,1.00)$ & 0.55 & $(-0.43,1.53)$ & 0.19 & $(-0.68,1.06)$ & 0.76 & $(-0.35,1.86)$ \\
\hline Parietal WM & -0.50 & $(-1.40,0.39)$ & -0.77 & $(-1.91,0.37)$ & -0.50 & $(-1.51,0.51)$ & -0.73 & $(-2.02,0.56)$ \\
\hline Frontal lobe & 0.12 & $(-1.58,1.82)$ & -1.18 & $(-3.34,0.98)$ & 0.28 & $(-1.62,2.18)$ & -1.31 & $(-3.72,1.11)$ \\
\hline Frontal GM & 0.45 & $(-0.74,1.64)$ & 0.03 & $(-1.48,1.54)$ & 0.58 & $(-0.76,1.92)$ & 0.18 & $(-1.52,1.88)$ \\
\hline Frontal WM & -0.13 & $(-1.53,1.26)$ & -1.59 & $(-3.36,0.18)$ & -0.12 & $(-1.68,1.45)$ & -1.76 & $(-3.75,0.24)$ \\
\hline Occipital lobe & -0.09 & $(-0.86,0.67)$ & 0.10 & $(-0.88,1.07)$ & -0.14 & $(-1.00,0.72)$ & 0.03 & $(-1.07,1.12)$ \\
\hline Occipital GM & -0.23 & $(-0.80,0.35)$ & 0.36 & $(-0.37,1.09)$ & -0.23 & $(-0.89,0.42)$ & 0.27 & $(-0.56,1.10)$ \\
\hline Occipital WM & 0.01 & $(-0.54,0.56)$ & -0.29 & $(-0.99,0.41)$ & -0.06 & $(-0.69,0.56)$ & -0.31 & $(-1.11,0.49)$ \\
\hline Hippocampus & -0.05 & $(-0.13,0.03)$ & 0.05 & $(-0.05,0.15)$ & -0.05 & $(-0.13,0.04)$ & 0.06 & $(-0.05,0.17)$ \\
\hline Entorhinal cortex & 0.00 & $(-0.01,0.01)$ & 0.00 & $(-0.01,0.02)$ & -0.01 & $(-0.02,0.01)$ & 0.01 & $(-0.01,0.02)$ \\
\hline Parahippocampal gyrus & 0.01 & $(-0.01,0.02)$ & 0.01 & $(-0.01,0.02)$ & 0.01 & $(-0.01,0.03)$ & 0.01 & $(-0.01,0.03)$ \\
\hline
\end{tabular}

Model 1 was a multivariable linear regression model adjusted by age, age-squared, intracranial volume, and sex. Model 2 was a multivariable linear regression model adjusted by age, age-squared, sex, educational level, alcohol consumption, intracranial volume, and vascular burden sum score categories $(0,1,2+$ cardiovascular risk factors). All continuous variables (age, intracranial volume, alcohol consumption, and pure-tone average) were mean-centered. Bolded values indicate that the $95 \%$ confidence interval does not overlap with 0 . The categories of auditory performance are: normal $(\leq-5.55 \mathrm{~dB})$, insufficient $(>-5.55$ to $\leq 3.80 \mathrm{~dB})$, and poor $(>-3.80 \mathrm{~dB})$.

GM indicates gray matter; WM, white matter.

(difference $=-0.211 \times 10^{-2}, 95 \% \mathrm{CI}:-0.0363 \times 10^{-2}$, $-0.0060 \times 10^{-2}$ ), while poor auditory performance was associated with lower FA (difference $=-0.0173 \times 10^{-2}$, $95 \%$ CI: $-0.0326 \times 10^{-2},-0.0020 \times 10^{-2}$ ) in Model 1 (Table 4). In Model 2, there were no associations of SRTdefined degrees of auditory performance with FA regardless of inclusion of PTA as an adjustment covariate.

\section{DISCUSSION}

Given the paucity of studies examining central auditory speech processing and its relationship to the brain, we evaluated the associations of central auditory speech processing, as assessed by SRTs on DIN tests, with brain volumes and WM microstructure after PTA adjustment in a population-based cohort study. When considering SRT as a continuous measure, poorer speech perception was associated with higher parietal lobe volumes, not with other brain volumes nor with DTI measures. Then, when using degrees of auditory performance to determine clinically meaningful categories, SRTdefined degrees of auditory performance were not associated with brain volumes. Insufficient auditory performance was associated with lower FA, while poor auditory performance was marginally associated with lower FA. These findings suggest associations of degrees of auditory performance with poorer WM microstructure. The lack of findings between central auditory function (speech comprehension after PTA adjustment) and brain volumes suggests that peripheral auditory function explains associations seen among unadjusted speech comprehension in noise, brain volumes, and WM microstructure. These findings highlight the differences in modeling continuous and categorical SRT in associations, and one type could be more informative than the other, depending on the research question.

When we used SRT as a continuous measure, poorer central auditory speech processing was associated with higher parietal lobe volumes. The parietal lobe plays a major role in semantic processing, and it is necessary for sound-based representations (30). We did not replicate findings from a previous study of 40 to 69 -year-old adults from the United Kingdom, Rudner et al. (31), which found that poorer central auditory speech processing, as defined by the DIN summary score, was associated with lower GM volumes. While both studies used DIN to define central auditory speech processing among participants of similar age range from the UK Biobank 
TABLE 4. Associations of speech recognition thresholds continuously and categorically with fractional anisotropy and mean diffusivity in the Rotterdam study $(n=2,226)$.

\begin{tabular}{|c|c|c|c|c|}
\hline & $\begin{array}{l}\text { Difference } \\
\text { in FA } \\
\text { (per 100) }\end{array}$ & $\begin{array}{c}95 \% \\
\text { Confidence } \\
\text { Interval }\end{array}$ & $\begin{array}{c}\text { Difference } \\
\text { in } \mathrm{MD} \\
\text { (per } 100 \mathrm{~m}^{2} / \mathrm{s} \text { ) }\end{array}$ & $\begin{array}{c}95 \% \\
\text { Confidence } \\
\text { Interval }\end{array}$ \\
\hline \multicolumn{5}{|l|}{ Continuous speech recognition thresholds } \\
\hline \multicolumn{5}{|l|}{ Model 1} \\
\hline Unadjusted by PTA & -0.0019 & $(-0.0036,-0.0002)$ & 0.0017 & $(-0.0008,0.0042)$ \\
\hline Adjusted by PTA & -0.0023 & $(-0.0047,0.0002)$ & 0.0021 & $(-0.0016,0.0057)$ \\
\hline \multicolumn{5}{|l|}{ Model 2} \\
\hline Unadjusted by PTA & -0.0012 & $(-0.003,0.0006)$ & 0.0012 & $(-0.0015,0.0040)$ \\
\hline Adjusted by PTA & -0.0012 & $(-0.004,0.0015)$ & 0.0021 & $(-0.0019,0.0061)$ \\
\hline \multicolumn{5}{|l|}{$\begin{array}{l}\text { Categorical speech recognition thresholds } \\
\text { Model } 1\end{array}$} \\
\hline \multicolumn{5}{|l|}{$\begin{array}{l}\text { Model } 1 \\
\text { Unadjusted by PTA }\end{array}$} \\
\hline Insufficient versus normal auditory performance & -0.0211 & $(-0.0363,-0.0060)$ & 0.0133 & $(-0.0090,0.0357)$ \\
\hline Poor versus normal auditory performance & -0.0173 & $(-0.0326,-0.0020)$ & 0.0133 & $(-0.0094,0.0360)$ \\
\hline \multicolumn{5}{|l|}{ Adjusted by PTA } \\
\hline Insufficient versus normal auditory performance & -0.0196 & $(-0.0354,-0.0039)$ & 0.0102 & $(-0.0130,0.0334)$ \\
\hline Poor versus normal auditory performance & -0.0186 & $(-0.0387,0.0014)$ & 0.0122 & $(-0.0174,0.0419)$ \\
\hline \multicolumn{5}{|l|}{ Model 2} \\
\hline \multicolumn{5}{|l|}{ Unadjusted by PTA } \\
\hline Insufficient versus normal auditory performance & -0.0150 & $(-0.0321,0.0021)$ & 0.0135 & $(-0.0118,0.0388)$ \\
\hline Poor versus normal auditory performance & -0.0124 & $(-0.0294,0.0047)$ & 0.0118 & $(-0.0135,0.0372)$ \\
\hline \multicolumn{5}{|l|}{ Adjusted by PTA } \\
\hline Insufficient versus normal auditory performance & -0.0132 & $(-0.0310,0.0046)$ & 0.0112 & $(-0.0151,0.0375)$ \\
\hline Poor versus normal auditory performance & -0.0129 & $(-0.0355,0.0096)$ & 0.0164 & $(-0.0170,0.0498)$ \\
\hline
\end{tabular}

Bolded values indicate $p<0.05$. Model 1 was a multivariable linear regression model adjusted by age, age-squared, and sex. Model 2 was a multivariable linear regression model adjusted by age, age-squared, sex, educational level, alcohol consumption, and vascular burden sum score categories $(0,1,2+$ cardiovascular risk factors). All continuous variables (age, alcohol consumption, and pure-tone average) were mean-centered. Bolded values indicate that the $95 \%$ confidence interval does not overlap with 0 . The categories of auditory performance are: normal $(\leq-5.55 \mathrm{~dB})$, insufficient $(>-5.55$ to $\leq 3.80 \mathrm{~dB})$, and poor $(>-3.80 \mathrm{~dB})$.

FA indicates fractional anisotropy; $\mathrm{MD}$, mean diffusivity; PTA, pure-tone average.

Study, Rudner et al. did not adjust the models by continuous PTA.

When we used SRT-defined categories to examine their associations with brain volumes, we observed associations of poor auditory performance with lower volumes in total brain and WM, especially in frontal and parietal WM when we did not adjust for PTA. In a previous cross-sectional study examining the association of PTA with brain volumes in the Rotterdam Study, Rigters et al. (32) found that poorer peripheral auditory function was associated with lower total brain volume and WM volume. When we did not adjust the analyses by PTA, we observed associations of poor auditory performance, as defined by SRT, with lower volumes in total brain and WM, particularly in frontal WM. These associations were no longer significant with the addition of PTA to the models, which suggests that these findings are more likely to be related to hearing ability than to the central aspects of listening in background noise. These findings also suggest that PTA should be included in models of central auditory speech processing and brain structure, to unravel central and peripheral auditory aspects.

Since hearing loss could be related to loss of WM microstructural integrity, we evaluated the associations of continuous SRT and SRT-defined degrees of auditory performance with global DTI measures. Rigters et al. (33) also evaluated the cross-sectional association of continuous SRT with global DTI measures in the Rotterdam Study. They observed that higher continuous SRT was associated with lower global FA, not global MD. Part of our results are consistent with this. In the model adjusted by age, age-squared, and sex, we found similar associations when defining central auditory speech processing either continuously or categorically. However, we did not observe an association after adjusting by vascular burden, education, and alcohol consumption, yet the direction of the relationship was the same between the two separate analyses. The differences in these associations could be due to the way vascular contributions were defined. Since vascular risk factors can co-occur with one another, we used a sum score, as opposed to the inclusion of each condition in the model. Moreover, the association between SRT and WM microstructure may be more regional than global. SRT was associated with lower FA in the uncinate fasciculus (33), an association tract that connects the limbic regions in the frontal lobe to the temporal lobe (34).

Main strengths of our study are the large populationbased sample with available hearing and neuroimaging assessments. There are also limitations of this study. First, this was a cross-sectional study, so we could not infer causality. Longitudinal data are needed to determine whether changes in brain volumes and white matter 
microstructure occur after the onset of hearing loss. Second, this study is generalizable to whites living in places similar to Ommoord, Rotterdam, The Netherlands, so the results might not be broadly applied to other populations, such as those of other ethnicities or in rural settings. Third, hearing aid use may have modified the associations that we observed, but we were underpowered to detect such associations. Approximately 5.7\% of the sample $(n=135)$ reported hearing aid use. Of these 135 individuals, the majority reported hearing aid use if they had moderate or greater hearing loss. Therefore, the distribution of hearing aid use was skewed toward individuals with worse hearing acuity.

In conclusion, we examined associations of both continuous and categorical SRT with brain volumes and global WM microstructure. While results differed by the way in which SRT was defined, this shows that degrees of auditory performance could provide more clinically relevant information regarding brain volumes and WM microstructure. As we showed, PTA is a major factor that can confound the relationship between central auditory processing and brain structure, so this is a factor to be included in models examining this question of interest. Furthermore, longitudinal studies are needed to determine how central auditory speech processing is associated with changes in brain volume and WM microstructure.

Acknowledgments: The authors thank all the participants of the study for time and Tekla Enser for performing the pure-tone audiometry and the speech-in-noise tests. This study is based on data collected in the context of the Rotterdam Study. The authors thank the Heinsius Houbolt Foundation for financial contribution to this study.

\section{REFERENCES}

1. Homans NC, Metselaar RM, Dingemanse JG, et al. Prevalence of age-related hearing loss, including sex differences, in older adults in a large cohort study. Laryngoscope 2017;127:725-30.

2. Livingston G, Sommerlad A, Orgeta V, et al. Dementia prevention, intervention, and care. Lancet 2017;390:2673-734.

3. Stenfelt S, Ronnberg J. The Signal-Cognition interface: Interactions between degraded auditory signals and cognitive processes. Scand $J$ Psychol 2009;50:385-93.

4. Pickles J. An Introduction to the Physiology of Hearing. Bingley, UK: Emerald Group Publishing; 2008.

5. Smits C, Kapteyn T S, Houtgast T. Development and validation of an automatic speech-in-noise screening test by telephone. Int $J$ Audiol 2004;43:15-28.

6. Smits C, Goverts ST, Festen JM. The digits-in-noise test: Assessing auditory speech recognition abilities in noise. $J$ Acoust Soc Am 2013;133:1693-706.

7. Ikram MA, Brusselle GGO, Murad SD, et al. The Rotterdam Study: 2018 update on objectives, design and main results. Eur J Epidemiol 2017;32:807-50.

8. de Bruijn RFAG, Bos MJ, Portegies MLP, et al. The potential for prevention of dementia across two decades: The prospective, population-based Rotterdam Study. BMC Med 2015;13:132.

9. Koole A, Nagtegaal AP, Homans NC, et al. Using the digits-in-noise test to estimate age-related hearing loss. Ear Hear 2016;37:508-13.

10. Dawes P, Fortnum H, Moore DR, et al. Hearing in middle age: A population snapshot of 40- to 69-year olds in the United Kingdom. Ear Hear 2014;35:e44-51.
11. Ikram MA, van der Lugt A, Niessen WJ, et al. The Rotterdam Scan Study: Design update 2016 and main findings. Eur J Epidemiol 2015;30:1299-315.

12. de Boer R, Vrooman HA, Van Der Lijn F, et al. White matter lesion extension to automatic brain tissue segmentation on MRI. Neuroimage 2009;45:1151-61.

13. Vrooman HA, Cocosco CA, van der Lijn F, et al. Multi-spectral brain tissue segmentation using automatically trained k-NearestNeighbor classification. Neuroimage 2007;37:71-81.

14. Sled JG, Zijdenbos AP, Evans AC. A nonparametric method for automatic correction of intensity nonuniformity in MRI data. IEEE Trans Med Imaging 1998;17:87-97.

15. Bokde AL, Teipel SJ, Schwarz R, et al. Reliable manual segmentation of the frontal, parietal, temporal, and occipital lobes on magnetic resonance images of healthy subjects. Brain Res Brain Res Protoc 2005;14:135-45.

16. Ikram MA, Vernooij MW, Hofman A, et al. Kidney function is related to cerebral small vessel disease. Stroke 2008;39:55-61.

17. Fischl B, Salat DH, Van Der Kouwe AJ, et al. Sequence-independent segmentation of magnetic resonance images. Neuroimage 2004:23:S69-84.

18. Armstrong NM, Huang C-W, Williams OA, et al. Sex differences in the association between amyloid and longitudinal brain volume change in cognitively normal older adults. Neuroimage Clin 2019;22:101769.

19. Driscoll I, Davatzikos C, An Y, et al. Longitudinal pattern of regional brain volume change differentiates normal aging from MCI. Neurology 2009;72:1906-13.

20. Galton CJ, Patterson K, Graham K, et al. Differing patterns of temporal atrophy in Alzheimer's disease and semantic dementia. Neurology 2001;57:216-25.

21. Du AT, Schuff N, Kramer JH, et al. Higher atrophy rate of entorhinal cortex than hippocampus in AD. Neurology 2004;62:422-7.

22. Convit A, de Asis J, de Leon MJ, et al. Atrophy of the medial occipitotemporal, inferior, and middle temporal gyri in nondemented elderly predict decline to Alzheimer's disease. Neurobiol Aging 2000;21:19-26.

23. Basser PJ, Mattiello J, LeBihan D. Estimation of the effective selfdiffusion tensor from the NMR spin echo. J Magn Reson B 1994; 103:247-54.

24. Basser PJ, Mattiello J, LeBihan D. MR diffusion tensor spectroscopy and imaging. Biophys $J$ 1994;66:259-67.

25. Koppelmans V, de Groot M, de Ruiter MB, et al. Global and focal white matter integrity in breast cancer survivors 20 years after adjuvant chemotherapy. Hum Brain Mapp 2014;35:889-99.

26. Pierpaoli C, Jezzard P, Basser PJ, et al. Diffusion tensor MR imaging of the human brain. Radiology 1996;201:637-48.

27. Gottesman RF, Schneider ALC, Zhou Y, et al. Association between midlife vascular risk factors and estimated brain amyloid deposition. JAMA 2017;317:1443-50.

28. Feunekes GI, Van Staveren WA, De Vries J, et al. Relative and biomarker-based validity of a food-frequency questionnaire estimating intake of fats and cholesterol. Am J Clin Nutr 1993;58:489-96.

29. Goldbohm RA, van den Brandt PA, Brants HA, et al. Validation of a dietary questionnaire used in a large-scale prospective cohort study on diet and cancer. Eur J Clin Nutr 1994;48:253-65.

30. Coslett HB, Schwartz MF. Chapter 18 - The parietal lobe and language. In: Vallar $G$, Coslett HB, eds Handbook of Clinical Neurology: Elsevier 2018;365-75.

31. Rudner M, Seeto M, Keidser G, et al. Poorer speech reception threshold in noise is associated with lower brain volume in auditory and cognitive processing regions. J Speech Lang Hear Res 2019;62: $1117-30$.

32. Rigters SC, Bos D, Metselaar M, et al. Hearing impairment is associated with smaller brain volume in aging. Front Aging Neurosci 2017;9:2.

33. Rigters SC, Cremers LGM, Ikram MA, et al. White-matter microstructure and hearing acuity in older adults: A population-based cross-sectional DTI study. Neurobiol Aging 2018;61:124-31.

34. Von Der Heide RJ, Skipper LM, Klobusicky E, et al. Dissecting the uncinate fasciculus: Disorders, controversies and a hypothesis. Brain 2013;136:1692-707. 